\title{
In Utero Bipolar Diathermy to Salvage a Phenotypically Normal Fetus in 45,X/46,XY Heterokaryotypic Monochorionic Twins*
}

\author{
Tuangsit Wataganara ${ }^{\# \#, ~ P o o m p o r n ~ U s c h a r a r a t t a n a s o p o n ~}{ }^{1}$, Chayawat Phatihattakorn", \\ Chanin Limwongse ${ }^{2}$, Sommai Viboonchart ${ }^{1}$, Katika Nawapun ${ }^{1}$ \\ ${ }^{1}$ Division of Maternal-Fetal Medicine, Department of Obstetrics and Gynecology, Faculty of Medicine, \\ Siriraj Hospital, Bangkok, Thailand \\ ${ }^{2}$ Division of Genetics, Department of Medicine, Faculty of Medicine, Siriraj Hospital, Bangkok, Thailand \\ Email: ${ }^{*}$ sitwg@mahidol.ac.th
}

Received November 6, 2011; revised December 4, 2011; accepted December 20, 2011

\begin{abstract}
Heterokaryotypic monozygotic twins are rare. We report a set of monochorionic diamniotic twins complicated with lethal malformations and hydrops in one fetus at menstrual age of 16 weeks'. Sonographic appearances and genetic amniocentesis result suggested for 45,X and 46,XY heterokaryotypic twins. Coexisting polyhydramnios found in the sac of severely malformed 45,X fetus threatened miscarriage and premature delivery. To salvage the phenotypically normal male fetus, the hydropic 45, X co-twin was selectively terminated by occlusion of its umbilical cord with bipolar diathermy. Complete cessation of funicular flow was achieved, resulting in normalization of total amniotic fluid volume. A healthy 2200-gram baby boy was born at 38 weeks'. His neurodevelopment was intact at 2 years of age. His physical examination was significant for slight growth delay (15 percentiles), with $6 \%$ of 45 ,X mosaicism found in his peripheral blood. Owing to the rarity of the cases, limited data is available regarding an optimal treatment for heterokaryotypic monochorionic twins with discordant lethal malformation. Intrauterine intervention may be offered should demise or permanent disabilities are imminent to the apparently normal fetus.
\end{abstract}

Keywords: Fetal Intervention; Heterokaryotypia; Monochorionic Twins; Bipolar Diathermy

\section{Introduction}

A set of monochorionic diamniotic twins was diagnosed during a sonographic examination at 12 menstrual weeks' in a 29-year-old, primigravidarum, Thai woman. Counterintuitively, one fetus was found to be male and the other was found to be female on her 16 weeks' scan. The female fetus showed signs of lethal hydrops, including generalized fluid collection and large, septated cystic hygroma. Echocardiographic findings of low insertion of tricuspid valve, ballooning right atrium, and large atrial septal defect suggest for Ebstein's anomaly. Pulse-wave Doppler interrogation demonstrated severe tricuspid regurgitation and reversed a-wave in every cardiac cycle. These Doppler findings, in the presence of fetal hydrops, suggested for cardiac decompensation [1]. Liquor volume in this amniotic sac was markedly increased, with deepest vertical pool of 12 centimeters. The male co-twin appeared structurally normal. The sonogram is shown in Figure 1.

Sonographic appearances suggested heterokaryotypic

*Conflict of interest: The authors declare no conflict of interest.

${ }^{\#}$ Corresponding author.
45,X/46, XY monochorionic twins. Other possibilities, such as $45, \mathrm{X} / 47, \mathrm{XYY}$, have also been reported [2]. Markedly increased liquor volume in the sac of severely malformed female fetus threatened miscarriage or preterm

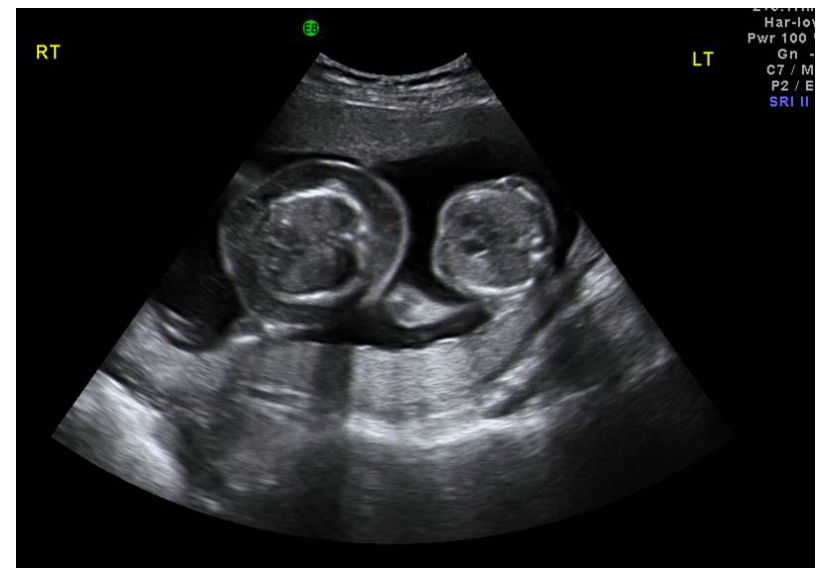

Figure 1. A set of heterokaryotypic monochorionic diamniotic twins at 16 weeks'. The female fetus was hydropic, while the male fetus appeared normal. 
delivery. Signs of early-onset cardiac decompensation suggest for a grave prognosis of this fetus. Currently, there are no fetal treatments available for Ebstein's anomaly. Therefore, plans were made to salvage the sonographically normal male fetus, by selectively terminate the female co-twin to normalize overall amniotic fluid volume. Genetic amniocentesis was performed only on the intendto-save male fetus to verify its karyotype for the merit of further intervention. It revealed a homogeneous 46,XY karyotype from 30 cells studied. The patient and her family were then extensively counseled for the seriousness of the condition and were offered treatment options including expectant management with timely delivery, termination of the whole pregnancy, serial amniodrainage, or selective termination of the malformed fetus. Umbilical cord occlusion with bipolar diathermy was chosen. This selective termination procedure due to lethal fetal malformations was approved by an abortion committee, according to the policy at our institute.

The procedure was performed at 19 weeks' after a written informed consent was obtained. The patient was premedicated with 10 milligrams of nifedipine and 1 gram of cefazolin. General anesthesia was used upon her request. It offers additional benefits of fetal analgesia and uterine relaxation. Sonographic mapping for the accessibility of the targeted umbilical cord was carried out. A 3.3-millimeter trocar (Karl Storz, Tuttlingen, Germany) was introduced through a small skin incision into the polyhydramniotic sac of the hydropic fetus under real-time ultrasound guidance. Twenty milliliters of amniotic fluid were initially obtained for chromosome studies. Bipolar forceps (Karl Storz, Tuttlingen, Germany) was then inserted to grab a free segment of the umbilical cord.

Electrical energy of 20 Watts was applied, with a gradual increment to the maximum of 40 Watts. This is to prevent precipitous vaporization of the water within the edematous Wharton's jelly, which can lead to cord rupture and fetal exanguination. Each coagulation episode lasted no longer than 60 seconds to avoid excessive heating in the amniotic cavity. Care was taken not to injure the cotwin, intertwin membranes, and uterine wall. Complete cessation of flow was confirmed by Doppler interrogation, as shown in Figure 2. Adjacent cord segment was coagulated in a similar fashion to ensure complete occlusion. Flow measurements were repeated to exclude the temporary obliteration as a result of vasospasm. One and a half liters of amniotic fluid was removed at the conclusion of the procedure. Operating time (trocar in and out) was 10 minutes. There was no funneling of internal cervical os or shortening of the cervical canal, therefore cervical cerclage was not placed. The patient was recovering in the hospital for the next 2 days. She was on ambulatory tocolysis with oral nifedipine for another week. Karyotype result of the hydropic fetus was homogeneous

\section{5,X from 21 cells studied.}

Her further prenatal care at a district hospital was uneventful. Continuous shrinkage of the 45,X sac was observed. She naturally delivered a healthy baby boy at 38 weeks', with the birth weight of 2200 grams (5th percentiles) [3]. Cord blood was not collected for chromosome study. Gross placental examination, as in Figure 3, showed monochorionic placenta with infarction in the 45,X territory. At 2 years of age, this boy had appropriate neurodevelopmental performances. Slight growth delay was observed (15 percentiles). Fluorescence in situ hybridization (FISH) studies from his peripheral blood showed $6 \%$ of 45,X mosaicism in 200 cultured lymphocyte colonies. Analysis from buccal mucosa revealed $1 \%$ of $45, \mathrm{X}$ mosaicism from 200 cells studied.

\section{Discussion}

This report presents a rare heterokaryotypia in monozygotic twins complicated with discordant lethal fetal

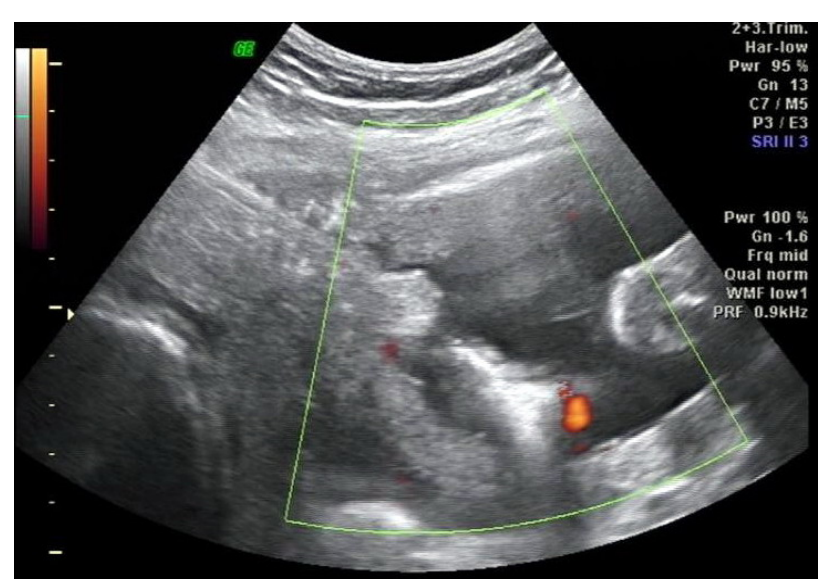

Figure 2. Bipolar diathermy of an umbilical cord segment of the $45, X$ fetus.

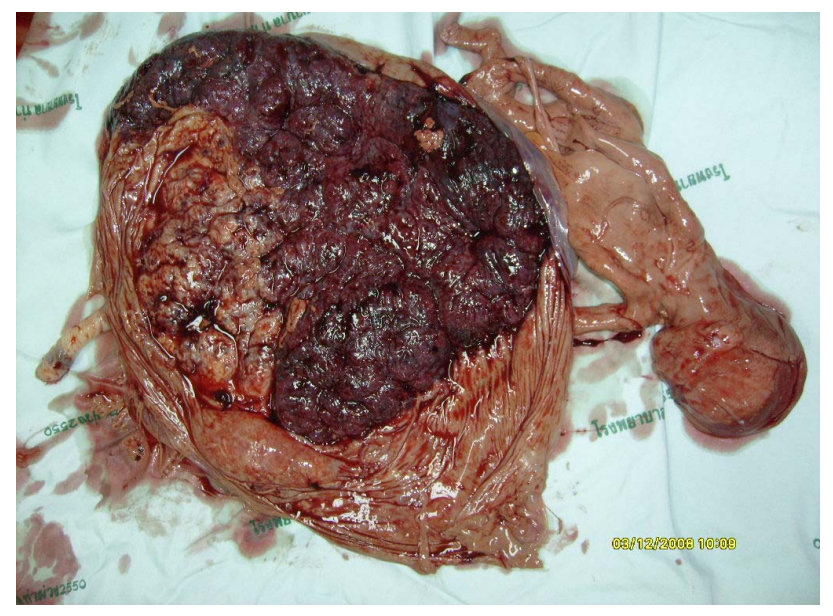

Figure 3. Placenta at the time of delivery showed a mummified 45, $\mathrm{X}$ fetus. Its placental territory was crumpled and encircled by the growing territory of 46,XY fetus. 
malformations and polyhydramnios. The aneuploidy fetus was unlikely to survive, and its increased liquor volume threatened the loss of the whole pregnancy. In addition, if the malformed fetus dies, there might be a passage of thromboplastic material or agonal hemodynamic changes through vascular anastomoses, leading to subsequent demise or necrotic cerebral lesions of its apparently normal co-twin $[4,5]$. This justified for the risks of a minimally invasive in utero procedure to salvage the other fetus. The most frequently reported form of heterokaryotypia is $45, \mathrm{X} / 46, \mathrm{XX}$, however, $45, \mathrm{X} / 46, \mathrm{XY}$ as in this presented case has also been described [6,7].

Strategic plans were arranged to limit the number of invasive procedures performed. Since the malformed fetus was unlikely to survive regardless of its karyotype, initial chromosome analysis was performed only from the phenotypically normal fetus. Rarer combination of $45, \mathrm{X} /$ 47,XYY may result in an anatomically normal male baby, with an increased risk of learning disabilities, behavioral difficulties, and infertility [8]. This possibility needs to be discussed with the parents, and shall be excluded prior to the surgically salvage therapy. Karyotype of the anomalous fetus can subsequently be confirmed during the surgery.

Selective termination of in discordant lethal malformation has been widely practiced [9]. Many interventions, such as injection of cardioplegic substances, coil embolization, and intraumbilical injection of air embolus or sclerosis agents, are not suitable in monochorionic twins. Laser dichorionization of the placenta can protect the normal fetus should its anomalous co-twin dies, but it does not stop the progressive polyhydramnios that threaten the whole pregnancy [10]. Transabdominal removal of abnormal fetus, so called sectio parva, has not been widely practiced due to its invasiveness [11]. Therefore, surgical vascular occlusion of the anomalous fetus is the most reasonable option. It can be accomplished using laser photocoagulation of the umbilical vessels, fetoscopic-assisted umbilical cord ligation, extracorporeal umbilical ligation, bipolar diathermy of the umbilical cord, interstitial laser ablation, or radiofrequency ablation.

Most experiences of surgical umbilical cord occlusion cluster around the treatment of twins reversed arterial perfusion sequence (TRAPS) [12]. Both funicular and intrafetal/interstitial approaches have been described. Randomized controlled trials for the best techniques may not be materialized due to the rarity of these conditions. This presented case shares some similarities with TRAPS, that the malformed fetus with virtually grave prognosis puts its healthy co-twin at risk. Umbilical cord of acardiac fetus tends to be short and edematous, making intrafetal approach technically less demanding than funicular approach. On the contrary, umbilical cord of this 45 , $\mathrm{X}$ fetus was long and mobile, therefore bipolar diathermy was chosen.
Possible explanations for low birth weight in this baby boy include placental infarction, chronic abruption, or chromosomal mosaicism. Persistent growth restriction, but not developmental delay, onto his childhood period suggests mosaicism origin. Intertwin transfusion may not be a significant contributor to the pool of 45 , X cells at this age. Percentage of mosaicism could be varied among the tissue sources studied [13]. Confirmation of zygosity after delivery can be accomplished using multiplex quantitative fluorescence polymerase chain reaction testing for DNA polymorphism on specific autosomes [14]. Growth of the surviving fetus after selective termination of its monochorionic co-twin has never been properly addressed. His future fertility function depends on the proportion of aneuploid cells in his testicles. Potential long-term consequences of survivors from heterokaryotypic multifetal gestation should be discussed with the family irrespective of fetal interventions.

\section{REFERENCES}

[1] T. Tongsong, P. Chanprapaph, S. Khunamornpong and S. Sirichotiyakul, "Sonographic Features of Ebstein Anomaly Associated with Hydrops Fetalis: A Report of Two Cases," Journal of Clinical Ultrasound, Vol. 33, No. 3, 2005, pp. 149-153. doi:10.1002/jcu.20102

[2] C. Bohec, N. Douet-Guilbert, A. Basinko, M. J. Le Bris, P. Marcorelles, M. P. Audrezet, et al., "Difficult Diagnosis and Management of an Heterokaryotypic Monochorionic Twin Pregnancy with Discordant Fetal Sex and 45,X/47,XYY Karyotypes,” Fetal \& Pediatric Pathology, Vol. 29, No. 6, 2010, pp. 424-430. doi:10.3109/15513815.2010.505630

[3] S. G. Babson and G. I. Benda, "Growth Graphs for the Clinical Assessment of Infants of Varying Gestational Age,” Journal of Pediatric, Vol. 89, No. 5, 1976, pp. 814820. doi:10.1016/S0022-3476(76)80815-3

[4] L. Fusi and H. Gordon, "Twin Pregnancy Complicated by Single Intrauterine Death. Problems and Outcome with Conservative Management,” British Journal of Obstetrics and Gynaecology, Vol. 97, No. 6, 1990, pp. 511-516. doi:10.1111/j.1471-0528.1990.tb02521.x

[5] S. S. Ong, J .Zamora, K. S. Khan and M. D. Kilby, "Prognosis for the Co-Twin Following Single-Twin Death: A Systematic Review,” BJOG: An International Journal of Obstetrics \& Gynaecology, Vol. 113, No. 9, 2006, pp. 992-998. doi:10.1111/j.1471-0528.2006.01027.x

[6] J. H. Edwards, T. Dent and J. Kahn, "Monozygotic Twins of Different Sex," Journal of Medical Genetics, Vol. 3, 1966, pp. 117-123. doi:10.1136/jmg.3.2.117

[7] T. Costa, M. Lambert, I. Teshima, P. N. Ray, C. L. Richer and L. Dallaire, "Monozygotic Twins with 45,X/46,XY Mosaicism Discordant for Phenotypic Sex," American Journal of Medical Genetics, Vol. 75, No. 1, 1998, pp. 40-44.

doi:10.1002/(SICI)1096-8628(19980106)75:1<40::AIDAJMG9>3.0.CO;2-U 
[8] M. Geerts, J. Steyaert and J. P. Fryns, "The XYY Syndrome: A Follow-Up Study on 38 Boys," Genetic Counseling, Vol. 14, No. 3, 2003, pp. 267-279.

[9] H. Vandecruys, K. Avgidou, E. Surerus, N. Flack and K. H. Nicolaides, "Dilemmas in the Management of Twins Discordant for Anencephaly Diagnosed at $11+0$ to $13+$ 6 Weeks of Gestation," Ultrasound in Obstetrics \& Gynecology, Vol. 28, No. 5, 2006, pp. 653-658. doi:10.1002/uog.2836

[10] L. Lewi, "Monochorionic Diamniotic Twin Pregnancies Pregnancy Outcome, Risk Stratification and Lessons Learnt from Placental Examination," Verh Koninklijke Academie voor Geneeskunde van België, Vol. 72, 2010, pp. 5-15.

[11] J. P. Bruner, T. C. Wheeler and M. J. Bliton, "Sectio Parva for Fetal Preservation," Fetal Diagnosis and Therapy, Vol. 14, No. 4, 1999, pp. 254-256.

doi:10.1159/000020932
[12] L. Lewi, C. Valencia, E. Gonzalez, J. Deprest and K. H. Nicolaides, "The Outcome of Twin Reversed Arterial Perfusion Sequence Diagnosed in the First Trimester," American Journal of Obstetrics and Gynecology, Vol. 203, No. 3, 2010, pp. 213.e1-213.e4.

[13] B. Gilbert, C. Yardin, S. Briault, V. Belin, A. Lienhardt, Y. Aubard, et al., "Prenatal Diagnosis of Female Monozygotic Twins Discordant for Turner Syndrome: Implications for Prenatal Genetic Counseling,” Prenatal Diagnosis, Vol. 22, No. 8, 2002, pp. 697-702. doi:10.1002/pd.383

[14] C. P. Chen, S. R. Chern and W. Wang, "Rapid Determination of Zygosity and Common Aneuploidies from Amniotic Fluid Cells Using Quantitative Fluorescent Polymerase Chain Reaction Following Genetic Amniocentesis in Multiple Pregnancies," Human Reproduction, Vol. 15, No. 4, 2000, pp. 929-934. doi:10.1093/humrep/15.4.929 